\title{
BULK METALLIC GLASS NANOWIRES FOR USE IN DIRECT ALCOHOL FUEL CELL Marcelo Carmo ${ }^{1 a}$, Shiyan Ding ${ }^{1 b}$, Golden Kumar ${ }^{1 b}$, Kai Sun ${ }^{2}$, Jan Schroers ${ }^{1 b}$ and André D. Taylor ${ }^{1 a^{*}}$ \\ ${ }^{1 \mathrm{a}}$ Chemical Engineering Department, \\ ${ }^{1 b}$ Mechanical Engineering Department \\ Yale University, New Haven, CT, USA \\ ${ }^{2}$ Department of Materials Science and Engineering - The University of Michigan, Ann Arbor, MI, USA
}

\section{ABSTRACT}

A key issue for direct alcohol fuel cells is its meager performance due to poor efficiency and durability of the catalysts. Developing a new class of materials that can outperform conventional catalysts during long-term operation is of critical importance. Here we report for the first time a nanoimprinting approach using $\mathrm{Pt}_{57.5} \mathrm{Cu}_{14.7} \mathrm{Ni}_{5.3} \mathrm{P}_{22.5}$ bulk metallic glass (BMG) to create a new class of high performance nanowire catalyst. Accelerated durability tests reveal that BMG nanowires maintain their electrochemical surface area in comparison to conventional Pt/C catalysts. BMGs represent a new class of high performance fuel cell catalysts that are CMOS compatible.

\section{INTRODUCTION}

Micro fuel cells were once championed as viable alternatives over existing battery technology for portable electronic devices. However, a key remaining issue is the meager performance of these devices due to poor efficiency and durability of the catalysts [1]. The poor durability of the commercial platinum supported on carbon black $(\mathrm{Pt} / \mathrm{C})$ catalyst is reflected by a significant loss of platinum electrochemical surface area (ECSA) over time during fuel cell operation [2]. The mechanisms for the loss of platinum ECSA (Fig. 1) have been discussed [2] and can be summarized as; corrosion of the carbon support, platinum dissolution and platinum nanoparticle aggregation.

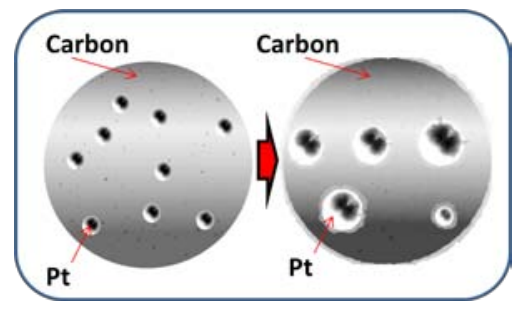

Figure 1 - Conventional Pt/C catalysts suffering from nanoparticles agglomeration and dissolution over long term operation.

Developing a new class of materials that can circumvent Pt-based anode poisoning (loss of efficiency/performance) and the agglomeration or dissolution (durability issue) of supported catalysts during longterm operation is of critical importance (Fig. 1). Here we report for the first time a CMOS compatible approach using $\mathrm{Pt}_{57.5} \mathrm{Cu}_{14.7} \mathrm{Ni}_{5.3} \mathrm{P}_{22.5}$ bulk metallic glass (BMG) [3] to create a new class of high performance nanowire fuel cell catalyst. These BMG nanowires materials have the potential to eliminate the degradation pathways described above. Since these catalysts do not require a support, the support corrosion problem is eliminated; BMG nanowires are also less vulnerable to dissolution during fuel cell operation than the $\mathrm{Pt} / \mathrm{C}$ nanoparticles. Additionally, the BMGs have an anisotropic morphology that can improve mass transport and catalyst utilization [4].

\section{RESULTS AND DISCUSSION}

Accelerated fuel cell durability comparisons reveal that BMG nanowires maintain their electrochemical surface area (ECSA) in comparison to conventional $\mathrm{Pt} / \mathrm{C}$ catalysts (Fig. 2). In fact, the
BMG ECSA showed a slight increase whereas the commercial $\mathrm{Pt} / \mathrm{C}$ catalyst lost about 35\% after 1000 cycles. This loss can be attributed to the agglomeration and dissolution of platinum nanoparticles (Fig. 1), while the increase in ECSA for the BMG represents an alloy improvement described by an increase in the Pt surface area as the copper in the BMG dissolves [5].

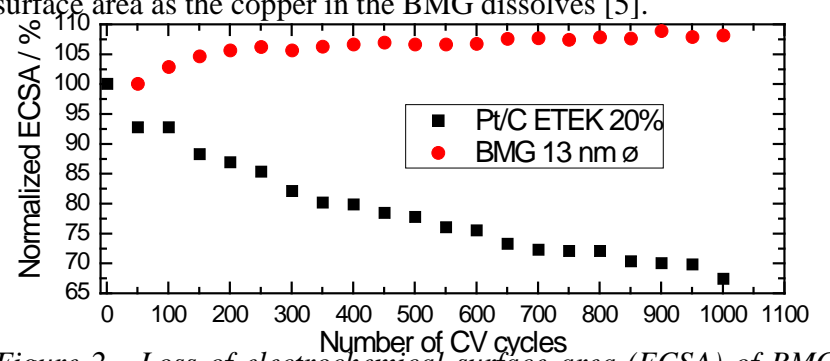

Figure 2 - Loss of electrochemical surface area (ECSA) of BMG $13 \mathrm{~nm}$ and Pt/C (E-TEK) catalysts with number of CV cycles in nitrogen-purged $0.5 \mathrm{molL}^{-1} \mathrm{H}_{2} \mathrm{SO}_{4}$ solution at room temperature (0.05-1.2 V vs. RHE, sweep rate $50 \mathrm{mVs}^{-1}$ ). The BMG catalyst maintains performance after 1000 cycles while the conventional ETEK catalyst decreases by $\sim 35 \%$.

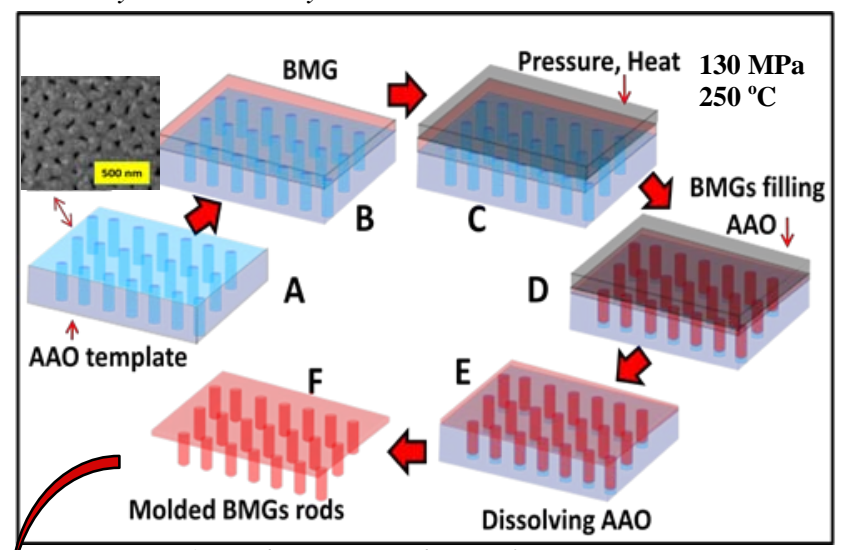

Figure 3 - Schematic synthesis of BMG nanowires.

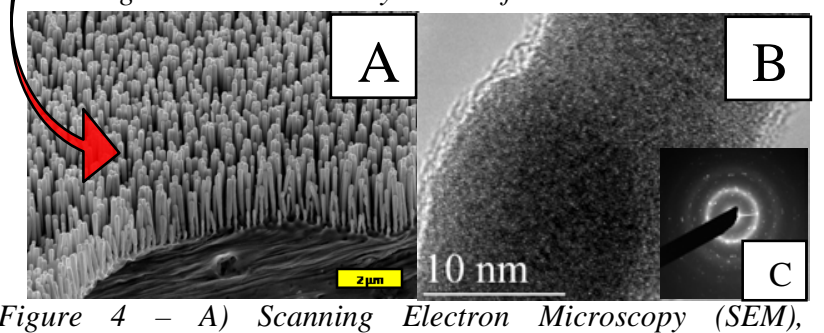

Figure 4 - A) Scanning Electron Microscopy (SEM), B) Transmission Electron Microscopy (TEM) and C) Selected Area Electron Diffraction (SAED) images for BMG nanowires.

BMGs are a subset of glass forming alloys that can be easily vitrified and formed into relatively large amorphous sections [3]. The absence of crystallites, grain boundaries and dislocations in the amorphous structure of bulk metallic glass results in a homogeneous and isotropic material down to the atomic scale, which displays very high strength, hardness, elastic strain limit and 
corrosion resistance. Because of their accommodation to dimensions at multiple length scales, BMGs can be formed into high catalyst surface areas without the need for a high surface area support. The BMG nanowire fabrication sequence (Fig. 3) allows the diameter of nanowires to be controlled from ca. $100 \mathrm{~nm}$ to 10 $\mathrm{nm}$ (Fig. 4) with an aspect ratio up to 200:1 [4]. These length scales are very close to conventional fuel cell supported catalyst particles dimensions and can easily be synthesized inside of a micro fabrication foundry, differentiating this process from existing complex catalyst synthesis techniques. The BMG is heated $\left(250{ }^{\circ} \mathrm{C}\right)$ into the super cooled liquid region where it softens and under applied pressure (130 MPa) can be thermoplastically imprinted (Fig. 3c) so that the BMG fills the nanopores (Fig. 3d). We note that the diameters of the BMG nanowires can be made even smaller dependent upon the template dimensions [3]. The alumina (AAO) template is dissolved in $\mathrm{KOH}$ solution (Fig. 3e) to isolate the nanowires. Fig. 4 shows the a) SEM and b) TEM image of the BMGs. The uniform vertical nanowires are well-isolated and parallel to one another. The selected area diffraction (SAED) image (Fig. 4c) suggests a polycrystalline Pt structure with a complete alloy formation, however, we are currently investigating whether the e-beam could influence the crystallinity of the BMG. Noble metal alloys have shown enhanced performance over conventional $\mathrm{Pt} / \mathrm{C}$ catalysts $[5,6]$, however, traditional thin film sputtering or e-beam evaporation cannot form the high surface areas needed for the electrochemical reactions $[5,7,8]$. Nanowires synthesized from organometallic precursors suffer from complex synthesis and low throughput/reproducibility (size control) [7]. Fig. 5a illustrates the $\mathrm{CO}$ oxidation for several electrodes used for this study: pure Pt, BMG flat surface, and BMG nanowires ( $\mathrm{x}$ length and diameters ranging from 100 down to $13 \mathrm{~nm}$.). The BMG nanowires have a higher $\mathrm{CO}_{\mathrm{ads}}$ tolerance due to a higher onset potential for $\mathrm{CO}$ oxidation.
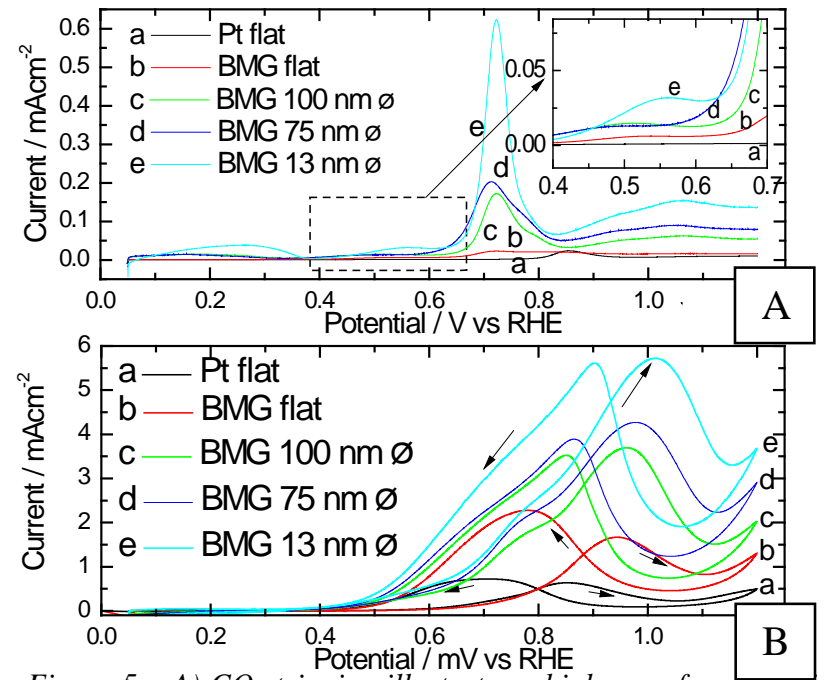

Figure $5-A)$ CO stripping illustrates a higher performance for adsorbed $\mathrm{CO}$ oxidation for BMGs over pure Pt. The wire diameters illustrate the higher surface area (performance) of the smaller diameter wires in the same fixed area and wire length. B) Ethanol $1.0 \mathrm{molL}^{-1}$ oxidation illustrating a superior catalyst performance of BMG materials for direct ethanol fuel cells in comparison to pure Pt. Electrolyte: $0.5 \mathrm{molL}^{-1} \mathrm{H}_{2} \mathrm{SO}_{4}$ solution at room temperature, sweep rate $50 \mathrm{mVs}^{-1}$.

This suggests that BMG nanowires are not as susceptible to self-poisoning (due to $\mathrm{CO}$ generation from the alcohol electrooxidation step) as pure platinum. Electrochemical characterizations show a higher ethanol activity for BMG nanowires (Fig. 5b).
The nanowires become more active at higher potentials $(\mathrm{E}>-0.2 \mathrm{~V})$ and demonstrate higher alcohol oxidation current densities than the BMG flat disc and pure Pt disc. The Pt-enriched nature of the outer surface enables all surface sites of the BMG nanowires to be highly conductive. This is conducive to the reaction kinetics on the catalyst surfaces, and hence may result in an enhancement in the electrochemical activity (Fig. 6).

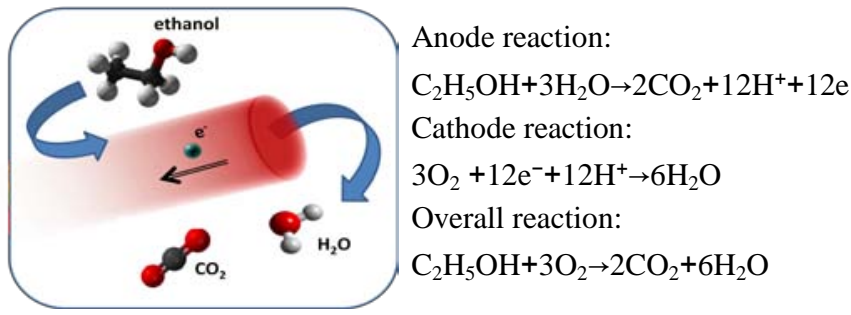

Figure 6 - Ethanol oxidation over bulk metallic glass (BMG) nanowire.

The electronic and strain effects may also play a major role in the activity enhancement. The enhanced activity for the alcohol oxidation Pt based BMGs with respect to pure Pt can also be ascribed to the synergetic effect of $\mathrm{Cu}$ and $\mathrm{Ni}$ oxides [1].

\section{CONCLUSIONS}

We have shown experimentally that the alcohol oxidation reaction activity and stability of fuel cell electrodes could be enhanced through the application of BMGs. BMG nanowires surpass the performance of conventional Pt/C catalysts. BMG nanowires created via nanoimprinting represent a new class of high performance fuel cell catalyst materials that are CMOS process compatible. On-going efforts are underway to integrate these materials into our previously described micro fuel cell architecture [8]. The performance can be further optimized through its chemical composition and structure to address a wide range of functionalities. The ability to form these materials into very high surface areas opens up a new range of possibilities for nextgeneration electrochemical devices and sensors.

\section{REFERENCES}

[1] K Kinoshita, Electrochemical oxygen technology, Wiley Inc., New York, 1992.

[2] P.J. Ferreira, et al., "Instability of Pt/C Electrocatalysts in PEMFC - A Mechanistic Investigation", Journal of the Electrochemical Society, 152, A2256, (2005).

[3] G. Kumar, H.X. Tang, J. Schroers, "Nanomoulding with Amorphous Metals", Nature, 457, 868 (2009).

[4] J. Shroers, N. Paton, "Amorphous Metal Alloys Form like Plastics", Advanced Materials \& Processes, 164, 61, (2006).

[5] P. Mani, R. Srivastava, P. Strasser, "Dealloyed Pt-Cu CoreShell Nanoparticle Electrocatalysts for Use in PEMFCs", Journal of Physical Chemistry C, 112, 2770 (2008).

[6] M. Carmo, et al., "Alternative Supports for the Preparation of Catalysts for PEMFCs: The Use of Carbon Nanotubes", Journal of Power Sources, 142, 169, (2005).

[7] J.T. Hu, T.W. Odom, C.M. Lieber, "Chemistry and Physics in One Dimension: Synthesis and Properties of Nanowires and Nanotubes", Accounts of Chemical Research, 32, 435, (1999).

[8] A.D. Taylor, et al., "Nanoimprinted Electrodes for Micro Fuel Cell Applications" Journal of Power Sources, 171, 218, (2007).

\section{CONTACT}

*André D. Taylor, tel: (203) 432-2217; andre.taylor@yale.edu 